\title{
La Interacción Mutua entre Fuentes y las Aparentes Anomalías en el Fenómeno de Interferencia
}

\author{
Reinaldo Welti \\ weltreb@arnet.com.ar \\ Facultad de Ciencias Exactas, Ingeniería y Agrimensura \\ Universidad Nacional de Rosario \\ Avda. Pellegrini 250 - (2000) Rosario - Argentina
}

Recebido em 31 de julho, 2002. Aceito em 16 de setembro, 2002.

\begin{abstract}
En la mayoría de los textos de física del nivel básico universitario al tratar el fenómeno de interferencia suponen que las fuentes irradian de la misma forma cualquiera sea la distancia entre ellas. En algunas situaciones esta suposición puede llevar a aparentes anomalías en la conservación de la energía. En este trabajo se muestra que estas anomalías se resuelven solamente si el fenómeno de interferencia se presenta en un contexto que tenga en cuenta las interacciones entre las fuentes y el generador que suministra energía a las mismas.

In almost all Physics texts of basic university level that treat the interference phenomenon it is supposed that sources irradiate in the same way whatever the distance was among them. In some situations this supposition leads to apparent anomalies in the conservation of the energy. In this work we demonstrate that these anomalies are solved only if the interference phenomenon is presented in a context that keeps in mind the interactions between the sources and the generator which gives the energy.
\end{abstract}

\section{Introducción}

Es bien conocido [1] que la intensidad de la radiación emitida por dos fuentes idénticas que oscilan con la misma amplitud, frecuencia y fase, es, en algunas direcciones, cuatro veces mayor que la que produciría una sola de las fuentes. En algunos textos [2] se comenta este hecho afirmando que "...sin embargo, como es claro, la energía total emitida por las dos fuentes es sólo el doble de la que emite una sola fuente. El aumento en un factor de más de dos en algunas direcciones se compensa por la existencia de intensidades cero en otras direcciones, a lo largo de las líneas nodales. La interferencia es esencialmente una distribución de le energía disponible"

Sin embargo, si las dos fuentes están alimentadas en fase, las líneas nodales desaparecen cuando la distancia entre fuentes $(d)$ es menor que la mitad de la longitud de onda $(\lambda)$. En el límite $d / \lambda \rightarrow 0$ la intensidad de la onda en todas las direcciones del espacio y, por lo tanto, la potencia total que emiten las dos fuentes es cuatro veces la potencia que emite cada una de las fuentes por separado. ¿Se ha creado energía? ¿De dónde proviene esta energía adicional?.

Cuando un radiador está operando en las proximidades de otros radiadores, el funcionamiento de cada fuente puede ser afectada por sus interacciones mutuas.
Una investigación cuantitativa de esta interacción requiere un conocimiento detallado de las perturbaciones que produce un radiador en los puntos en los que se encuentran los radiadores vecinos y de las perturbaciones que estos producen en la zona en la que se encuentra el primero.

$\mathrm{Al}$ estudiar la interferencia de la radiación emitida por dos fuentes se supone que la "amplitud de la oscilación" de las fuentes (la corriente en los conductores de una antena que emite ondas de radio, o la velocidad de las membrana en un parlante que emite ondas sonoras) es independiente de la distancia entre las fuentes. Sin embargo, como veremos en la próxima sección, para que no se altere la amplitud de la oscilación cuando se varía la distancia entre las fuentes, la potencia que el generador suministra a los emisores debe modificarse. En particular, si las fuentes están alimentadas en fase y $d / \lambda \rightarrow 0$, el generador debe duplicar la potencia que entrega a cada emisor.

La interacción mutua entre fuentes es importante cuando la distancia entre ellas es comparable o menor que la longitud de onda. En la mayoría de los dispositivos que se utilizan para estudiar la interferencia de las ondas electromagnéticas en el rango visible, la distancia entre fuentes es mucho más grande que la longitud de onda y la interacción mutua entre las fuentes puede ser despreciada. Esto podría explicar el motivo por el 
cual en el nivel básico, que usualmente restringe la interferencia a las ondas luminosas, ignora por completo la interacción mutua.

En los últimos años se han obtenido resultados experimentales [3][4] sobre la interferencia de la luz que pasa a través de una formación de pequeños orificios, practicados sobre láminas de oro y separados por distancias comparables con la longitud de onda. En estos experimentos se observaron algunas aparentes anomalías en la intensidad de la luz difractada por estos orificios, que podrían explicarse si se tienen en cuenta las interacciones mutuas entre los mismos.

En el amplio rango de frecuencias de las ondas de radio la interacción mutua entre antenas es un fenómeno bien conocido. En estos casos la interacción mutua entre las antenas se describe mediante las así llamadas $\mathrm{im}$ pedancias mutuas [5]. El cálculo de estas impedancias mutuas es, en general, muy complejo, pues el método que se utiliza requiere el conocimiento de los campos en la zona cercana.

La interacción mutua entre emisores de ondas acústicas (parlantes) ha sido analizado recientemente [6] utilizando un método sugerido por Pritchard en 1960 [7] que requiere sólo el conocimiento del campo en la zona lejana. Esto facilita la complejidad de los cálculos involucrados. No tenemos conocimiento que este método "acústico" haya sido utilizado para el cálculo de impedancias mutuas entre antenas.

\section{Interferencia de dos fuentes alimentadas en fase}

En la Fig. 1 se muestra un sistema de dos fuentes idénticas $S_{1}$ y $S_{2}$, ubicadas a lo largo del eje $z$ y separadas por una distancia $d$. Las fuentes están excitadas con la misma frecuencia, amplitud y fase. La intensidad de la onda $I$ (joule $/ \mathrm{m}^{2} \mathrm{~s}$ ) irradiada por este sistema, en el punto $P$ de coordenadas $(r, \theta, \phi)$, que se muestra en la Fig. 1, viene dada por [8] :

$$
I(\theta, \phi)=4 I_{1}(\theta, \phi) \cos ^{2}\left(\frac{k d}{2} \cos \theta\right)
$$

donde

$$
I_{1}(\theta, \phi)=I_{0} G(\theta, \phi)
$$

es la intensidad emitida por cada una de las fuentes mientras que $I_{0}$ es la intensidad que crea en el punto $P$ un radiador isotrópico que irradia la misma potencia que cada una de las fuentes por separado. Si $W_{0}$ es esta potencia, $I_{0}=W_{0} / 4 \pi r^{2}$. La función $G(\theta, \phi)$ es la función directividad de cada una de las fuentes. Si las fuentes son isotrópicas $G(\theta, \phi)=1$. Observemos que de acuerdo a estas definiciones la integral de $G(\theta, \phi)$ sobre todo el ángulo sólido que rodea a $O$ es $4 \pi$.
Los puntos del espacio en los cuales $I(\theta, \phi)=0$, (superficie nodal) satisfacen la ecuación

$$
\cos \theta= \pm(2 n+1) \frac{\lambda}{2 d}
$$

cualquiera sea $\phi$. Estas superficies son conos. Si $d \gg \lambda$, hay muchas superficies nodales. Entre dos superficies nodales se tienen superficies sobre las cuales la intensidad es $4 I_{0}$. Por lo tanto, la intensidad promediada sobre todas las direcciones es $2 I_{0}$ y la potencia total emitida es la suma de las potencias de las dos fuentes.

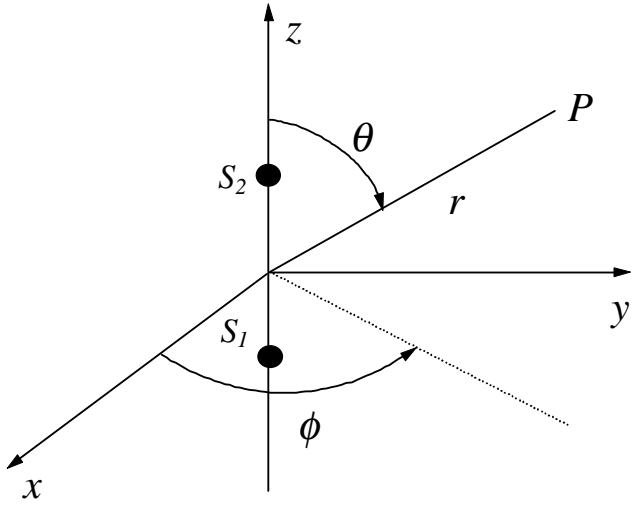

Figura 1. Interferencia entre dos fuentes isotrópicas.

Sin embargo, como lo menciona Crawford [8], cuando las fuentes están separadas por una distancia $d<\lambda / 2$ no hay más superficies nodales en el diagrama de radiación y el promedio de $I$ es mayor que $2 I_{0}$. En el caso límite en el que $d / \lambda \rightarrow 0$, la ecuación (1) se reduce

$$
I \approx 4 I_{0} G(\theta, \phi)
$$

En este caso, la potencia total no es la suma de las potencias entregadas por las dos fuentes sino el doble de esta suma. ¿Es esta una violación del principio de conservación de la energía? No, para nada. En realidad, como veremos, cada fuente emite dos veces más energía en presencia de la otra.¿Cómo es posible esto? En la deducción de la ecuación (1) está implícita la suposición de que el "movimiento" de las fuentes es independiente de la distancia entre las mismas. Sin embargo, como veremos en la próxima sección, para que no cambie el "movimiento" de los emisores el generador que alimenta las fuentes debe duplicar la potencia que entrega a cada una de ellas.

\section{Impedancia mutua de dos emisores}

Para fijar ideas supondremos que los radiadores son antenas dipolo alimentadas en su punto medio como se 
muestra en la Fig. 2. Los dos dipolos tienen iguales dimensiones, son paralelos y están separados por una distancia $d$. Aplicamos al primer dipolo la tensión $V_{1}$ de frecuencia $\omega$, y al segundo dipolo, la tensión $V_{2}$ de igual frecuencia y fase. En los dipolos se originan corrientes eléctricas, cuyas amplitudes complejas en los terminales de entrada se designan por $I_{1}$ e $I_{2}$, respectivamente.

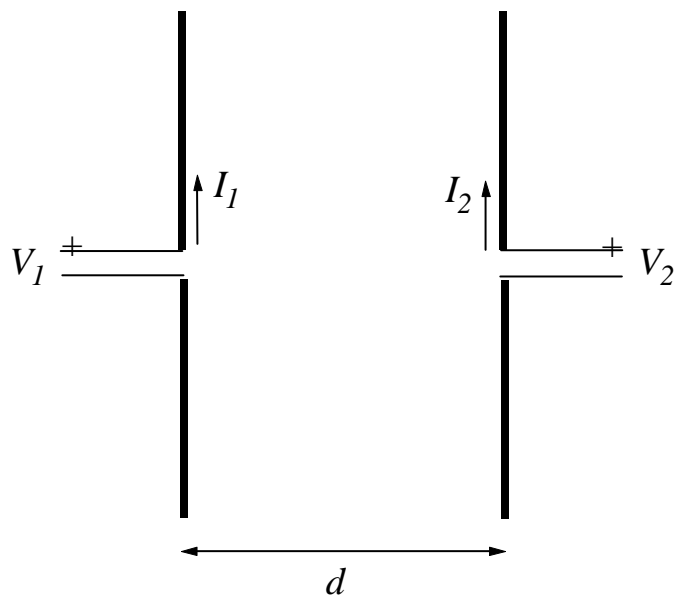

Figura 2. Dos antenas dipolos paralelas con acoplamiento mutuo.

El diagrama de radiación del sistema de dos dipolos depende esencialmente de la relación entre las amplitudes y las diferencias de fase de las corrientes $I_{1}$ e $I_{2}$ en sus entradas, las que dependen, a su vez, de las tensiones $V_{1}$ y $V_{2}$ aplicadas en esos terminales. En teoría de circuitos, la aplicación del teorema de reciprocidad ${ }^{1}$ permite deducir las siguientes relaciones entre las tensiones y las corrientes de entradas:

$$
\begin{aligned}
& V_{1}=Z_{11} I_{1}+Z_{12} I_{2} \\
& V_{2}=Z_{21} I_{1}+Z_{22} I_{2}
\end{aligned}
$$

donde $Z_{11}$ y $Z_{22}$ son las impedancias propias de las antenas 1 y 2 , mientras que $Z_{12}$ y $Z_{21}$ son sus impedancias mutuas. Estas impedancias mutuas tienen en cuenta las interacciones entre las dos antenas. La corriente $I_{1}$ de la antena 1 crea un campo eléctrico en la zona que la rodea y este campo eléctrico induce una fem $Z_{12} I_{1}$ en la antena 2. De la misma manera la corriente de la antena 2 induce la fem $Z_{21} I_{2}$ en la antena 1. En la ecuación (2) las tensiones y las corrientes son generalmente complejas. El cálculo de las impedancias mutuas es en general muy complicado [9].

Mediante el teorema de reciprocidad se demuestra que:

$$
Z_{21}=Z_{12}
$$

Como los dos dipolos son idénticos tenemos también que $Z_{11}=Z_{22}$. Remarquemos, sin embargo, que la igualdad (3) es válida aún cuando las antenas sean diferentes.

Si las antenas están alimentadas con la misma tensión, $V_{1}=V_{2}$, entonces por simetría, $I_{1}=I_{2}$, y por lo tanto:

$$
V_{1}=\left(Z_{11}+Z_{12}\right) I_{1}
$$

Para calcular la potencia media $W_{1}$ que el generador entrega a la fuente 1 (la misma cantidad le entrega a la fuente 2) se multiplica (4) por $I_{1}^{*}$ y se calcula posteriormente la parte real

$$
W_{1}=\operatorname{Re}\left(\frac{1}{2} V_{1} I_{1}^{*}\right)=\frac{1}{2}\left\|I_{1}\right\|^{2}\left(R_{11}+R_{12}\right)
$$

Esta expresión puede escribirse de la forma:

$$
W_{1}=W_{0}\left(1+\frac{R_{12}}{R_{11}}\right)
$$

donde

$$
W_{0}=\frac{1}{2} R_{11}\left\|I_{1}\right\|^{2}
$$

es la potencia que absorbe la antena 1 en ausencia de la antena $2, R_{11}$ es la denominada "resistencia de radiación" de la antena 1 , mientras que $R_{12}$ es la parte real de la impedancia mutua $Z_{12}$. En ausencia de pérdidas, la potencia que absorbe la antena, es la potencia que ésta irradia. La potencia total $W$ que irradian las dos fuentes es, por lo tanto, $W=W_{1}+W_{2}=2 W_{1}$, esto es:

$$
W=2 W_{0}\left(1+\frac{R_{12}}{R_{11}}\right)
$$

Observemos que la potencia $W$ que irradian las dos fuentes no es la suma de las potencias que irradian cada una de ellas cuando actúan por separado. Esto se debe a la interacción mutua entre las fuentes, que en la ecuación (8) está representada por la resistencia mutua $R_{12}$. En la sección anterior apuntamos que cuando la distancia entre las fuentes, es muy grande comparada con la longitud de onda, los valores máximos de la intensidad de la onda radiada $\left(4 I_{0}\right)$ se alternan con los nulos en numerosas direcciones del espacio, dando un valor medio para $I$ de $2 I_{0}$. Por lo tanto, la potencia emitida por las dos fuentes es $2 W_{0}$. Esto significa que $R_{12}$ debe hacerse cero, cuando $d / \lambda \rightarrow \infty$, lo que es razonable, pues si las distancias entre las antenas o fuentes es muy grande (medidas en longitudes de ondas) la interacción mutua entre las mismas debe ser muy pequeña. Si la distancia entre las antenas es mucho menor

\footnotetext{
${ }^{1}$ El teorema de reciprocidad afirma que "... en todo sistema compuesto de impedancias lineales bidireccionales, si se aplica un voltaje $V$ entre cualquier par de puntos y se mide la corriente $I$ en cualquier rama, la razón $V$ a $I$ llamada impedancia transferida, será la misma que la razón $V$ a $I$ que se obtiene cuando las posiciones del generador y del amperímetro se intercambian".
} 
que la longitud de onda, la intensidad de la onda es $4 I_{0}$ en todas las direcciones del espacio y por lo tanto la potencia irradiada por las dos fuentes es $4 W_{0}$. De acuerdo con la ecuación (8) esto implica que $R_{12} \rightarrow R_{11}$ cuando $d / \lambda \rightarrow 0$. La ecuación (6) nos dice que en esta situación, la potencia que el generador entrega a cada una de las fuentes se duplica. En conclusión, cuando $d / \lambda \rightarrow 0$ la potencia total emitida es cuatro veces la potencia que emite cada una de ellas por separado.

Argumentos similares pueden utilizarse si en lugar de dos antenas que emiten ondas electromagnéticas se tienen dos radiadores (parlantes) que emiten ondas sonoras como se muestra en la Fig. 3. Cuando un parlante está operando en las proximidades de otro, el funcionamiento de cada unidad es afectada por sus interacciones mutuas.

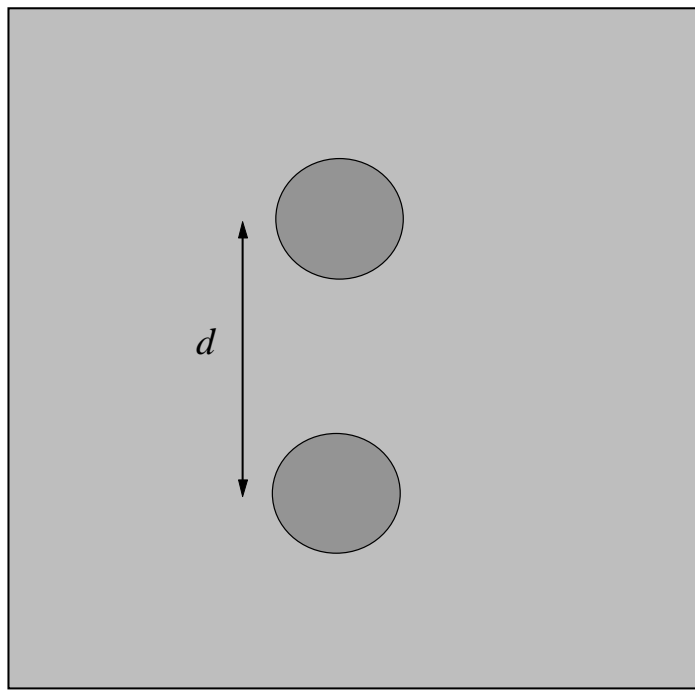

Figura 3. Dos parlantes con acoplamiento mutuo.

Consideremos el sistema de dos radiadores planos (circulares de radio $a$ ) que están insertados en una pantalla (baffle) rígida infinita. Si designamos con $f_{1}$ y $f_{2}$ las fuerzas que actúan sobre cada uno de los radiadores y con $v_{1}$ y $v_{2}$ la velocidad de sus superficies, se demuestra [9] de manera análoga al caso eléctrico, que están relacionadas a través de las ecuaciones :

$$
\begin{aligned}
& f_{1}=Z_{11} v_{1}+Z_{12} v_{2} \\
& f_{2}=Z_{12} v_{2}+Z_{22} v_{2}
\end{aligned}
$$

Las impedancias $Z_{12}$ y $Z_{21}$ son las impedancias mutuas entre los radiadores 1 y 2 , mientras que $Z_{11}$ y $Z_{22}$ son las autoimpedancias de los radiadores 1 y 2 respectivamente. Si los radiadores son idénticos y si las fuerzas que actúan sobre ellos son las mismas $f_{1}=f_{2}$, entonces por simetría $v_{1}=v_{2} \mathrm{y}$, por lo tanto:

$$
f_{1}=\left(Z_{11}+Z_{12}\right) v_{1}
$$

La potencia media irradiada por el radiador $1, W_{1}$ se obtiene multiplicando (10) por $v_{1}^{*} / 2$ y luego calculando la parte real:

$$
W_{1}=\operatorname{Re}\left(\frac{1}{2} f_{1} v_{1}^{*}\right)=\frac{1}{2}\left\|v_{1}\right\|^{2}\left(R_{11}+R_{12}\right)
$$

De la misma manera que para el caso de las dos antenas de ondas de radio se puede concluir que la resistencia mutua $R_{12} \rightarrow 0$ cuando $d / \lambda \rightarrow \infty$ y que $R_{12} \rightarrow R_{11}$ cuando $d / \lambda \rightarrow 0$.

Si las "fuentes" son dos orificios practicados sobre una superficie conductora o dieléctrica, existirá una interacción mutua entre los dos orificios cuando se los ilumina con un haz de luz coherente, pero la descripción y cuantificación de la misma es más compleja que los dos casos que analizamos más arriba.

\section{Cálculo de la resistencia mu- tua en función de $d / \lambda$}

Se puede calcular la resistencia mutua, para cualquier valor de la distancia entre las fuentes, siempre que se conozca la función $G(\theta, \phi)$, esto es, el diagrama de radiación de cada una de las fuentes.

La potencia media total $W$ que irradia el sistema de dos radiadores se obtiene integrando (1) a través de una superficie esférica $S$, de un radio $r$ muy grande, con centro en el punto medio de los dos radiadores. Muy grande significa que la esfera está en la zona de radiación $\left(r \gg d^{2} / 2 \lambda\right)$.

$$
W=\int 4 I_{0} G(\theta, \phi) \cos ^{2}\left(\frac{k d}{2} \cos \theta\right) r^{2} d \Omega
$$

donde $d \Omega$ es el diferencial de ángulo sólido. Si utilizamos la igualdad trigonométrica

$$
\cos ^{2}(\alpha / 2)=\frac{1+\cos \alpha}{2}
$$

la ecuación (12) puede reescribirse de la forma

$$
W=2 W_{0}\left(1+\frac{1}{W_{0}} \int I_{0} G(\theta, \phi) \cos (k d \cos \theta) r^{2} d \Omega\right)
$$

donde

$$
W_{9}=\int I_{0} G(\theta, \phi) r^{2} d \Omega
$$

es la potencia que irradiaría cualquiera de las dos fuentes en la ausencia de la otra.

Si no hay pérdidas toda la potencia que entrega el generador a las fuentes es irradiada por las mismas. Por lo tanto, si comparamos (8) con (13) obtenemos que 


$$
\frac{R_{12}}{R_{11}}=\frac{1}{W_{0}} \int I_{0} G(\theta, \phi) \cos (k d \cos \theta) r^{2} d \Omega
$$

Si las dos fuentes son isotrópicas $I_{0}=W_{0} / 4 \pi r^{2}$, entonces

$\frac{R_{12}}{R_{11}}=\frac{1}{4 \pi} \int \cos (k d \cos \theta) d \Omega=\frac{1}{2} \int_{0}^{\pi} \cos (k d \cos \theta) \operatorname{sen} \theta d \theta$

Esta última integral es inmediata y se obtiene

$$
\frac{R_{12}}{R_{11}}=1+\frac{\operatorname{sen} k d}{k d}
$$

Si el radio $a$ de los parlantes de la Fig. 1 es mucho menor que la longitud de onda $\lambda$, cada uno de los parlantes se comporta como un radiador isotrópico y los resultados obtenidos por Scandrett et al (2001) coinciden con la ecuación (16).

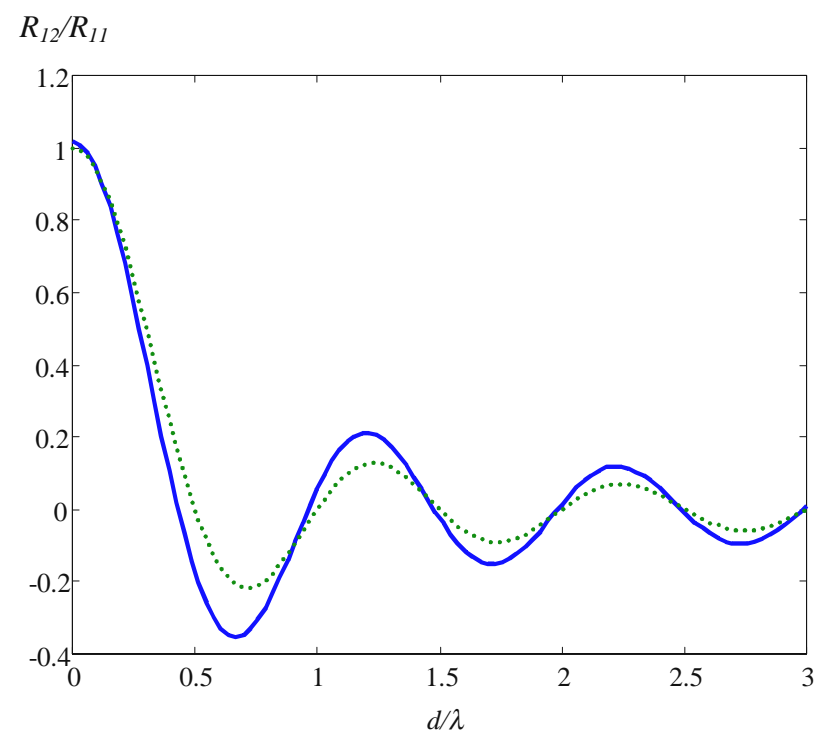

Figura 4. Gráficas de $R_{12} / R_{11}$ en función de $d / \lambda$. En líneas llenas para un sistema de dosdipolos de media onda y en líneas punteadas para dos radiadores isotrópicos.

La ecuación (16), válida para radiadores isotrópicos, puede resultar aproximadamente válida para radiadores no "muy" directivos ("cuasi-isotrópicos") como las antenas dipolo de media onda. En la Fig. 4 se muestra en líneas llenas la gráfica de $R_{12} / R_{11}$ para un dipolo de media onda que se calcula mediante el método de la "fem inducida" [10] y en líneas de puntos la gráfica de la ecuación (16).

Si se introduce en la ecuación (14) la directividad de una antena dipolo de media onda se obtiene una expresión para la resistencia mutua muy parecida a la que se obtiene con el método tradicional de la fem inducida. El cálculo de las integrales de la ecuación (14) es, sin embargo, mucho más simple y además puede utilizarse para el cálculo de la impedancia mutua para otros tipos de antenas que no son abordables con el método tradicional.

\section{Interferencia de dos fuentes en oposición de fase}

Si las dos fuentes de la Fig. 3 oscilan en oposición de fase la intensidad de la radiación emitida viene dada por

$$
I(\theta, \phi)=4 I_{1}(\theta . \phi) \operatorname{sen}^{2}\left(\frac{k d}{2} \cos \theta\right)
$$

Si $d \gg \lambda$, el promedio de $I(\theta, \phi)$ sobre todas las direcciones del espacio es aproximadamente igual a $2 I_{0}$, que es la suma de las intensidades que irradian cada una de las fuentes por separado. Sin embargo, si $d / \lambda \rightarrow 0$, $I(\theta, \phi) \rightarrow 0$ en todas las direcciones del espacio. Se explica habitualmente este resultado diciendo que las ondas emitidas por las dos fuentes llegan en oposición de fase en cualquier punto del espacio y se produce una interferencia destructiva. La amplitud de la onda y, por lo tanto, la intensidad de la misma es cero en todos los puntos del espacio. Ante esta situación la pregunta habitual es la siguiente: ¿qué ocurre con la energía asociada con cada una de las dos ondas?. En esta pregunta está implícita la suposición absurda de que la energía se destruye.

Cuando las dos fuentes están en oposición de fase la ecuación (8) se transforma en

$$
W=2 W_{0}\left(1-\frac{R_{12}}{R_{11}}\right)
$$

mientras que la (14) para $R_{12} / R_{11}$ es la misma. Cuando $d / \lambda<<1, R_{12} \rightarrow R_{11}$ y, por lo tanto, $W \rightarrow 0$. El generador no entrega energía a las fuentes. La energía que el generador le entrega a una de las fuentes es absorbida por la otra y no existe onda irradiada. La energía no se destruye.

\section{Conclusión}

Al ignorar las fuentes y sumideros de las ondas, el análisis de la interferencia puede llegar a resultados contradictorios. Las ondas son creadas por fuentes y éstas interactúan entre sí. La física de esta interacción es muy diferente en los distintos casos: en las antenas, la corriente de una de ellas genera un campo eléctrico y éste induce una fem en la otra; en los parlantes, el movimiento de la membrana de uno de ellos genera una variación de presión que produce una fuerza sobre la membrana del otro. En los pequeños orificios practicados sobre una superficie de metal, el mecanismo físico de la interacción está aún en discusión [11], algunos lo atribuyen a los plasmones superficiales y otros a las ondas de Bloch. 
Si la interacción entre las fuentes es lineal, siempre se puede encontrar un sistema de ecuaciones - entre las variables apropiadas del problema particular - similares a las ecuaciones (2) y (9). El hecho remarcable es que la impedancia mutua que interviene en estas ecuaciones, puede ser deducida del diagrama de interferencia. El fenómeno de interferencia es formalmente el mismo para todos los tipos de ondas: las expresiones matemáticas que la describen dependen de la geometría, la longitud de onda, la orientación relativa y el diagrama de radiación de las dos fuentes y no del tipo de ondas que éstas emiten. La interacción mutua entre las fuentes que se deriva del diagrama de interferencia tiene, por lo tanto, un cierto carácter de "universal". Por este motivo, las dos curvas de la Fig. 4, que representan las impedancias mutuas de dos fuentes sonoras isotrópicas y de dos antenas dipolo que emiten ondas de radio, son muy similares y la pequeña diferencia se debe a que la antena de radio de media onda no es isotrópica.

\section{Referências}

[1] Tipler P. A. Física, Editorial Reverté, S.A. 1995

[2] French, A. P. (1982). Vibraciones y Ondas, Editorial Reverté, Barcelona.

[3] Ghaemi H., Thio T., Grupp D.E., Ebbesen T.W., y Lezec H.J. (1998 ). "Surface plasmons enhance optical transmission trough subwavelength holes", Phys. Rev. B 58 (11), 6779-6782.

[4] Sönnichsen C., Duch A., Tae Jin Kim G., Thio T., Ebbesen T., Grupp D. y Lezec H. (1999). "Control of optical transmission trough metals perforated with subwavelength arrays", Optical Letters, 24 (4), pp. 256-258.

[5] Collin, R. (1985). Antennas and Radiowave Propagation, McGraw-Hill, Inc, New York

[6] Scandrett, C. , Day J. y Baker S.. (2001). "A modal Pritchard approximation for computing array element mutual impedance", J. of Acoustic Soc. Am., 109 (6), 2001, $2715-2729$.

[7] Pritchard, R. L. (1960) "Mutual acoustic impedance between radiators in an infinite rigid plane", J. Acoustic Soc. Am., 32, 730-737.

[8] Crawford, F.(1975). Ondes, Cours de Physique, Vol. 3, Librairie Armand Colin, Paris.

[9] Kinsler, L., Frey, A., Coppens, A., Sanders, J. (1982). Fundamental Acoustics. John Wiley \& Sons. New York.

[10] King, R. W. P., Mack R. y Sandler S. (1968). Arrays of Cylindrical Dipole Antennas, Cambridge University Pres, London.

[11] Bains, S. High transmission through subwavelenght gratings inspires debate. OE-Reports, Technology and Trends for the International Optical Enginneering Community, 195, (2000). http:// www.spie.org/web/oer/march/mar00/transmission.html 